\title{
Renal Infarction: Three Case Reports and Literature Review
}

\author{
Luis Sepulveda $^{\mathrm{a}, \mathrm{c}}$, Manuel Oliveira ${ }^{\mathrm{b}}$, Alcino Oliveira ${ }^{\mathrm{a}}$, Joaquim Mendes ${ }^{\mathrm{a}}$, Filipe Rodrigues ${ }^{\mathrm{a}}$
}

\begin{abstract}
The renal infarction is a rare condition with nonspecific and often similar clinical presentation to other more common renal diseases such as urinary lithiasis. Early diagnosis and treatment is crucial to prevent irreversible renal damage and recurrence of thromboembolic events. The most common causes are embolic heart disease, renal trauma and atheromatous disease. However, in some rare cases the etiology isn't clear (idiopathic renal infarcts). The authors present three cases of renal infarction with similar clinical presentation and therapeutic approach although distinct etiologies, evolution and prognosis.
\end{abstract}

Keywords: Renal infarction; Embolism; Atrial fibrillation; Renal artery aneurysm; Idiopathic

\section{Introduction}

The renal infarction is a rare disease, with an estimated incidence in Emergency Room (ER) of 0.004 to $0.007 \%$ [1-3]. In 1940, in a study involving more than 14,000 autopsies, Hoxie $\mathrm{H}$ and colleagues found 205 cases of renal infarction ( $1.4 \%$ prevalence) with only two cases being diagnosed during life. This study indicated that a high number of patients with renal infarction were not correctly diagnosed [4]. The nonspecific clinical presentation, often similar to renal colic or pyelonephritis, is one of the main factors responsible for error or delay in diagnosis, leading to prolonged ischemia

\footnotetext{
Manuscript accepted for publication December 20, 2013

${ }^{a}$ Urology Department of Centro Hospitalar de Tras-os-Montes e Alto Douro (CHTMAD), Portugal

${ }^{\mathrm{b}}$ Urology Department of Hospital de Santo Antonio - Oporto, Portugal

${ }^{\mathrm{c} C}$ Corresponding author: Luis Sepulveda, Urology Department of Centro Hospitalar de Tras-os-Montes e Alto Douro, Avenida da Noruega 5000-508 Vila Real, Portugal. Email: LuisSepulveda.Uro@gmail.com
}

doi: http://dx.doi.org/10.14740/wjnu120w time and development of irreversible renal damage $[1,5,6]$.

The most common causes of renal infarction are thromboembolism from cardiac origin (acute myocardial infarction, valvular heart disease, arrhythmias) and atheromatous disease $[7,8]$. However, there are other less frequent causes described in the literature such as hypercoagulable states (thrombophilia, sickle cell disease), cocaine abuse, renal trauma, neoplastic and renal vascular disease (such as fibromuscular dysplasia, vasculitis and dissecting aortic aneurysm) $[1,2,5,7,9]$. There are also sparse reports of renal infarction secondary to septic emboli in the context of systemic candidiasis or due to embolism in Takotsubo syndrome $[10,11]$. In some rare cases the etiology isn't clear, thus these are considered as idiopathic renal infarcts [7]. Characteristically these occur in younger patients and with fewer thromboembolic risk factors [8].

Although the study of Bolderman et al, involving 27 patients with renal infarction, demonstrated that only $41 \%$ presented heart disease, most authors still believe the main cause to be due to structural or functional cardiac pathology $[2,7,12,13]$. Furthermore it demonstrated that renal infarcts unrelated to cardiac pathology, similar to idiopathic infarction, occurred at earlier ages and with less cardiovascular risk factors [12].

The authors present three cases of renal infarcts with similar clinical presentation but distinct etiology and evolution.

\section{Case Report}

\section{Case 1}

Male patient of 46 years old referred to the ER for prolonged right back pain, with 26 hours of evolution, with no history of trauma, fever or hematuria. Except for hypertension and hypercholesterolemia the patient had no other comorbidities such as coagulation disorders, diabetes mellitus, renal or heart disease. He denied smoking or alcohol habits. Blood analysis revealed leukocytosis $\left(20.41 \times 10^{3} / \mu \mathrm{L}\right.$ with $85.8 \%$ neutrophils), slight elevation of transaminases (aspartate transaminase $114 \mathrm{U} / \mathrm{L}$, alanine transaminase $111 \mathrm{U} / \mathrm{L}$ ) and 

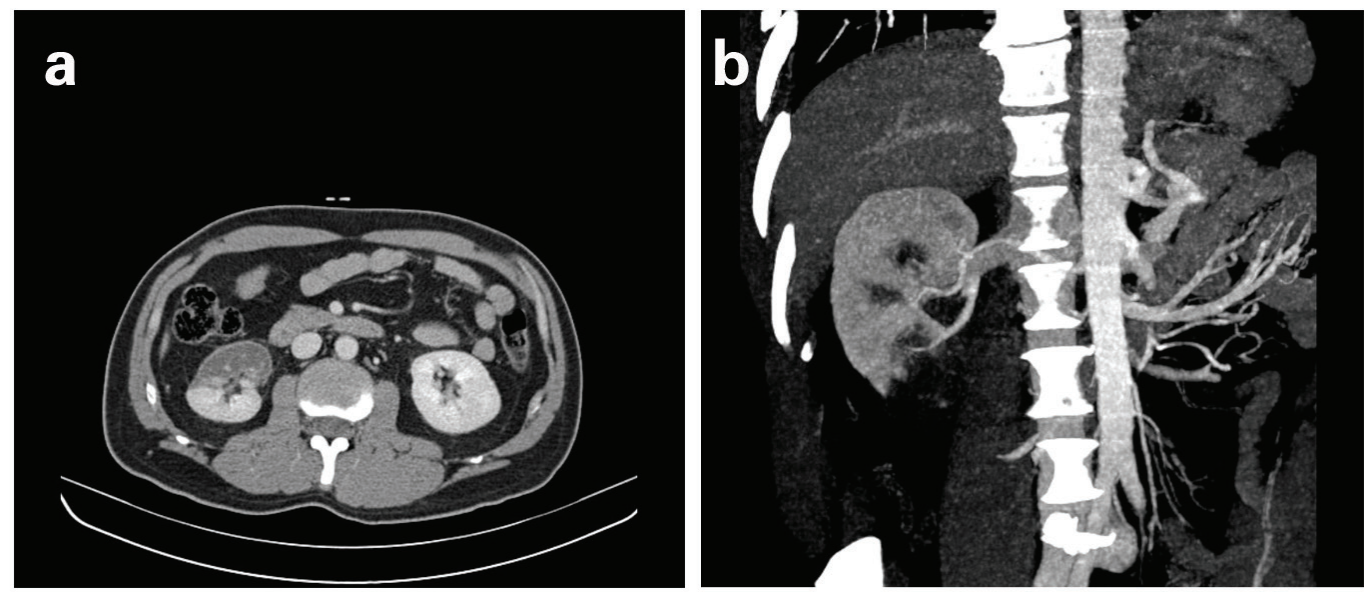

Figure 1. a) Contrast-enhanced CT showing non-enhanced areas of the anterior surface and lower pole of the right kidney, suggestive of renal infarction. b) Coronal reconstruction demonstrating the absence of contrast enhancement of the inferior pole of the right kidney.

frank elevation of lactate dehydrogenase (LDH) - $985 \mathrm{U} / \mathrm{L}$ (reference value 135 - $225 \mathrm{U} / \mathrm{L}$ ). Urine dipstick was normal. Abdominal ultrasound revealed liver steatosis and a five millimeters polyp in gallbladder, with no renal, spleen or pancreas abnormalities. Contrast enhanced computed tomography (CECT) demonstrated partial renal infarction concerning the anterior and inferior portions of the right kidney due to occlusion of the inferior branch of the right renal artery (Fig 1a, b). According to vascular surgery observation, given the time course and infarct location, there was no indication for invasive procedures. The patient was admitted for surveillance and started low molecular weight heparin and intravenous antibiotics.

The etiological study through electrocardiogram (ECG), echocardiography, genetic thrombophilia assay, evaluation of coagulation inhibitors and screening of anti-phospholipid syndrome showed no changes.

CT angiography confirmed the existence of occlusion of the lower branch of the anterior division of the renal artery with subsequent infarction of the inferior pole of the right kidney. The renal angiogram revealed multifocal infarction, absence of significant stenosis of the right renal artery and probable vascular malformation in a branch of the renal artery that, at the time, wouldn't justify intervention or the clinic presentation. The patient was discharged 7 days later on oral anticoagulation in therapeutic range (INR between two and three) and surveillance in Urology and Vascular Surgery consultation. The follow-up renal scintigraphy with Tc99m-DMSA performed two months later revealed atrophy of the right kidney, with reduced uptake of the drug and 38\% of
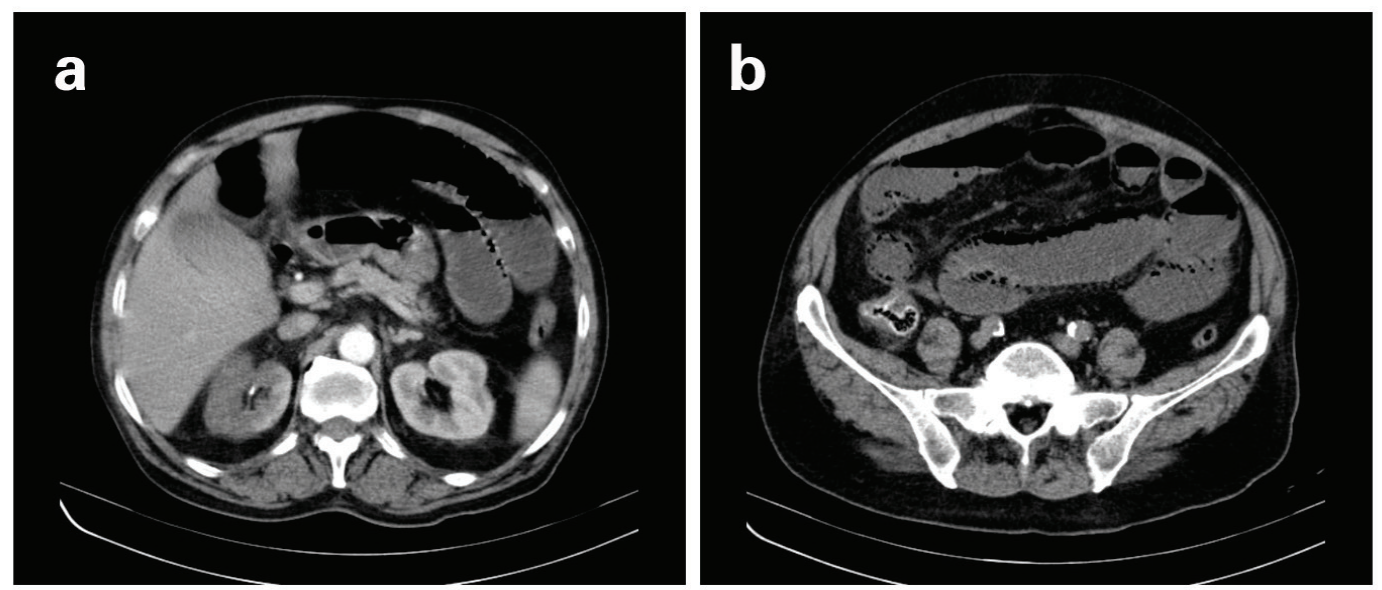

Figure 2. a) CECT revealing absence of contrast uptake by almost entire right kidney, suggesting large renal infarction. b) CECT revealing gas bubbles inside the wall of the small intestine loops, indicating ischemic bowel. 

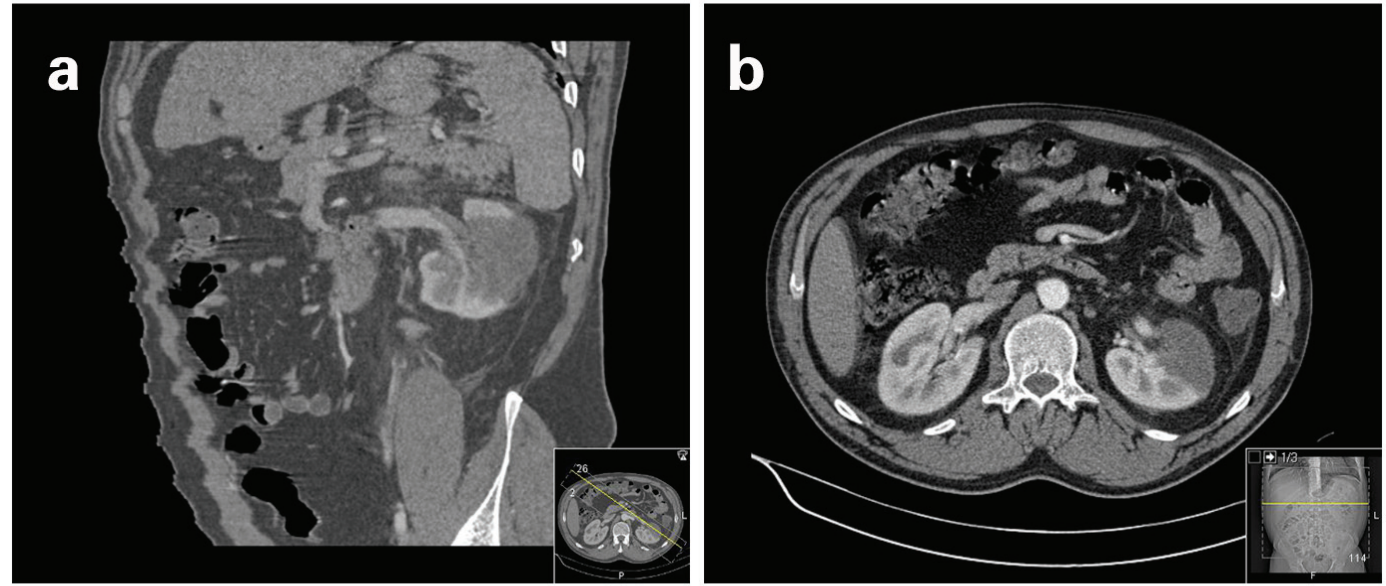

Figure 3. a) CT angiography reconstruction showing infarction of a large area of the left kidney due to occlusion of a left renal artery branch. b) Abdominal CT revealing absence of contrast enhancement of the anterior portion of the left kidney.

differential renal function for the right kidney and $62 \%$ for the left kidney. Re-evaluation with renal angiography in Vascular Surgery consultation revealed a saccular aneurysm of the right renal artery, most likely to be the primary cause of renal infarction. At 6 months follow-up the patient presented normal renal function and no relevant symptoms.

\section{Case 2}

Male patient of 79 years old referred to the ER for sudden onset of abdominal pain with several hours of evolution, with no relief position, located in the upper quadrant and right flank, associated with nausea and vomiting. The patient had multiple cardiovascular risk factors, including hypertension, history of heavy smoking (associated with chronic bronchitis), dyslipidemia, diabetes mellitus and congestive heart failure. Clinically the patient was very agitated, hypertensive (blood pressure of 190/96 $\mathrm{mmHg}$ ), afebrile and with pain at palpation of the right abdominal quadrant but without evidence of peritoneal irritation. The blood analysis revealed increased lactate dehydrogenase (LDH), 1,218 U/L, C-reactive protein (CRP) $39.7 \mathrm{mg} / \mathrm{dL}$ (reference values $0.0-0.5$ $\mathrm{mg} / \mathrm{dL}$ ), creatinine $1.7 \mathrm{mg} / \mathrm{dL}$ and urea $81 \mathrm{mg} / \mathrm{dL}$, with normal serum potassium and transaminases. Contrast-enhanced CT revealed low uptake of the entire right renal parenchyma and no excretion of contrast in this kidney secondary to obstruction at the distal portion of the right renal artery (Fig. 2a). It also showed extensive atheromatous plaque of upper and infrarenal abdominal aorta. According to Vascular Surgery's opinion, at that time there was no indication for interventional therapy. The patient was initiated in low molecular weight heparin at therapeutic dosage and later substitution to coumarin therapy.

In the following days, the patient remained hypertensive, controlled with labetolol and presented deterioration of renal function and onset of atrial fibrillation (AF) with fast ventricular response. At the third day, due to sudden aggravation of abdominal pain and peritoneal irritation, the patient repeated the CT study, which revealed probable bowel ischemia (Fig. $2 b)$. An emergency exploratory laparotomy confirmed the suspicion of bowel infarction and lead to resection of $70 \mathrm{~cm}$ of ischemic distal jejunum. In the first days after surgery the patient presented severe sepsis with multiple organ dysfunctions (including renal, respiratory and circulatory failure). Despite the poor prognosis the patient progressed favorably, clinically and analytically: renal function recovered gradually, with progressive reduction of inflammatory parameters, hemodynamic stability and reversion of atrial fibrillation to sinus rhythm. Echocardiography performed during hospitalization showed left ventricular hypertrophy with akinesia of various segments of the ventricular walls and moderate depression of left ventricular systolic function. There weren't any intracardiac thrombi detected. Three weeks after admission the patient was transferred to the Hospital nearest to his residence, where he was discharged a few days later. Presently the patient is on chronic coumarin therapy, with normal renal function and under surveillance on Cardiology consultation.

\section{Case 3}

A 35 years old man, smoker and without other cardiovascular risk factors, was transferred to the ER for evaluation by Urology on suspicion of left renal colic. Clinically the 
patient presented intense pain in left lumbar region radiating to the left lower abdominal quadrant. The non-enhanced CT revealed diffuse hepatic esteatosis, preserved kidney cortex and absence of dilatation of pyelocaliceal system. There was no evidence of renal or ureteral calculus bilaterally. Blood analysis presented leukocytosis $\left(16.6 \times 10^{3} / \mu \mathrm{L}\right)$ and no relevant alteration of renal parameters. LDH values weren't initially assessed. Urinary microscopy revealed sediment with phosphates amorphous, without leukocytes, erythrocytes or nitrites. The urine culture was negative. After observation by Surgery on suspicion of bowel colic the patient was medicated with anti-spasmodic, analgesics and discharged with indication for surveillance at home.

The patient was reassessed 2 days later for aggravation of algic complaints, refractory to the analgesics initially prescribed. Reevaluation of blood analysis presented normal hemogram and normal hepatic and renal function, although a slight elevation of LDH (398 U/L). CECT demonstrated absence of contrast uptake in the inferior pole and external surface of the left kidney due to the occlusion of a branch of the left renal artery (Fig. 3a, b). Given the course of evolution, the patient was initiated on low molecular weight heparin in therapeutic dosage (enoxaparin $80 \mathrm{mg}$ twice daily). The etiologic study by CT angiography, echocardiogram, ECG, auto-immune and prothrombotic assay (factor V Leiden, lupus anticoagulant, protein $\mathrm{C}$ and $\mathrm{S}$, anticardiolipin, antiphospholipid, antinuclear and antineutrophil cytoplasmatic antibodies) was negative. The genetic study for thrombophilia was also negative. The renal arteriography didn't reveal stenosis, dissection or aneurysm of the renal artery or its main branches, presenting however slow progression of contrast in the inferior pole and absence of uptake in the more peripheral segments. The patient progressed uneventfully, without algic complaints since the second day of admission. The patient was discharged at the 14th day treated with anti-platelet aggregation and guided to follow up in Urology consult.

\section{Discussion}

Usually the clinical presentation in renal infarction is nonspecific and overlapping with other more common entities such as infectious or lithiasic kidney disease [1,5]. The typical patient has an average age between 60 and 70 years, with abdominal or lower back pain, usually constant, accompanied by nausea, vomiting and fever $[1,3,6]$. Hypertension, frequently secondary to alterations of the renin-angiotensin system, can be a presenting sign for renal infarction $[8,14]$. Blood count commonly presents leukocytosis, elevated LDH (some studies describe as typical LDH values of four times higher than normal) and mild elevation of transaminases and C-reactive protein $[2,13,15]$. Gross hematuria is rare, although patients can frequently present microscopic hematuria [3]. Serum creatinine can also be high at presentation or suffer slight increase in the days following diagnosis, particularly in cases of solitary kidney or bilateral infarction [1, $3,16]$. Given the unspecific presentation and the importance of early diagnosis, it is essential to maintain a high degree of clinical suspicion in the presence of an acute lumbar and/or flank pain in a patient with thromboembolic risk factors and increased LDH [16].

In the study of Tsai SH et al involving 18 acute renal infarcts, the average time between the onset of symptoms and definitive diagnosis was 1.9 days, similar to other studies that revealed times between 2 and 336 hours [2, 3, 6, 7]. Given that normal kidney tissue tolerates complete arterial obstruction for 90 to 180 minutes until onset of irreversible lesions, it is essential to establish an early diagnosis for therapeutic revascularization and consequently to a better prognosis of the patient $[1,15]$.

Although most authors still consider angiography as the gold standard exam, the increased use of computed tomography (CT) in the ER significantly contributes to reduce the time to definitive diagnosis and the number of miss-diagnosed cases $[6,15]$. However, in the ER, the use of non-enhanced contrast CT is more common which, despite allowing diagnosing or excluding other causes of acute abdominal pain such as renal colic, appendicitis, diverticulitis, gynecological pathology, among others, lacks specificity and sensitivity for thromboembolic and renovascular disease [3]. Thus, the clinician should be aware of the importance of a contrast-enhanced CT in a patient with thromboembolic risk factors and clinical presentation suggestive of renal infarction, despite the presence of a previously normal nonenhanced CT study. The third case is an example of the low sensitivity of non-enhanced CT in the diagnosis of renal infarction and the importance of clinical suspicion in patients with inconclusive imaging study.

The renal infarct can have different presentations in the CT study: most commonly appears as a wedge-shaped perfusion defect, but it can also present as multifocal or global renal ischemia, rarely associated with mass effect [17]. It is also typical the presence of a cortical rim sign, an enhanced thin layer of renal parenchyma at the periphery, due to preserved capsular circulation $[3,8,17]$.

Etiological investigation is a fundamental aspect in the evaluation of renal infarction and should include the following exams: ECG (eventually with treadmill test), Holter, echocardiography, CT angiography and evaluation for prothrombotic and autoantibodies assay [12]. In most cases an early etiologic diagnosis allows to prevent recurrence of thromboembolic events, favoring the prognosis of the patient. Unfortunately, in Case 2, despite the early onset of anticoagulation therapy after diagnosis of extensive abdominal atheromatous plaque and atrial fibrillation, the patient presented a second thromboembolic event leading to bowel ischemia and deterioration of the patient's prognosis.

Given the rarity of this disease, there isn't enough evi- 
dence concerning therapeutic options, especially on the value of low molecular weight heparins, thrombolytic efficacy or comparative studies of medical versus surgical therapy [6, $7,13,16]$. Based on the consensus of the literature, anticoagulation should be initiated with heparin and later conversion to coumarin therapy. Parameters as the dosage of heparin, the target INR and the duration of oral anticoagulant therapy aren't well defined and should be adjusted case-to-case. INR values between two and three are the most recommended, maintained indefinitely if chronic prothrombotic conditions are present. In case of early diagnosis, bilateral lesion or a solitary kidney, the use of thrombolytics or surgical embolectomy should be considered $[1,7,13,16]$. This therapeutic modality is supported mainly in case descriptions, which reported good rates of reperfusion, although different renal prognosis [6-8]. Some authors consider it to be a viable option when performed within the first 12 hours of ischemia, despite the significant lower tolerance time of the kidney when subjected to total arterial occlusion [7]. In all three cases, the time until definitive diagnosis exceeded 24 hours, well above the estimated time for successful revascularization attempts by thrombolytics or thrombectomy.

According to most studies the prognosis and long-term outcome depends more on the underlying condition or comorbidities than to the renal infarction itself [18]. The Case 2 is an example, in which the presence of extensive atherosclerotic pathology and atrial fibrillation lead to infarction of other organs besides the kidney with aggravation of clinical status and poorer prognosis.

The most common sequels are renal failure and persistent hypertension $[6,8]$. Although most cases show a progressive improvement of renal function and resolution of hypertension over time, a small percentage may need to be included on a dialysis program $[3,8,14,19]$. In case of hypertension following renal infarction, angiotensin-converting enzyme (ACE) inhibitors or angiotensin receptor antagonists, are theoretically the most appropriate drugs given the likelihood of overactivation of renin-angiotensin system $[5,14,15]$.

According to Tsai et al, follow-up should include renal scintigraphy: evaluation of renal function based on serum creatinine or creatinine clearance rate is elusive due to the compensatory hypertrophy of the contralateral kidney [7].

The authors present three different cases of renal infarction: the first case reports a relatively young patient with few risk factors for thromboembolic events, whose angiography revealed a saccular aneurysm of the right renal artery, which could, in fact, be the cause of renal infarction. The second case is a more common example of an elderly patient with multiple cardiovascular risk factors, extensive atheromatous plaque of the abdominal aorta and atrial fibrillation with rapid ventricular response. Although both cases were promptly initiated with low molecular weight heparin at therapeutic dosage followed by oral anticoagulants (warfarin), the second case showed worse overall prognosis given the underly- ing pathology and recurrence of thromboembolic event. The third case describes a young patient with few cardiovascular risk factors and no apparent underlying cause, suggestive of idiopathic renal infarction.

\section{Key Points}

1) The clinical presentation in renal infarction is nonspecific and overlapping with other more common entities such as infectious or renal calculus disease.

2) It is essential to establish an early diagnosis to improve the long-term outcome.

3) A Contrast-enhanced CT should be performed in a patient with thromboembolic risk factors, clinical presentation compatible to renal infarction and with an apparently normal non-enhanced CT study.

\section{Acknowledgements}

This work was supported by Sanofi-Aventis.

\section{Conflict of Interests}

The authors have no conflict of interests.

\section{References}

1. Javaid MM, Butt MA, Syed Y, Carr P. Acute renal infarction: an unusual cause of abdominal pain. Ren Fail. 2009;31(5):406-408.

2. Cabral Ribeiro J, Sousa L, Calaza C, Santos A. Acute segmental renal infarction due to factor V Leiden. Arch Esp Urol. 2009;62(6):486-488.

3. Korzets Z, Plotkin E, Bernheim J, Zissin R. The clinical spectrum of acute renal infarction. Isr Med Assoc J. 2002;4(10):781-784.

4. Hoxie HJ, Coggin CB. Renal infarction statistical study of two hundred and five cases and detailed report of an unusual case. Arch Intern Med. 1940; 65:587-594.

5. Barbey F, Matthieu C, Nseir G, Burnier M, Teta D. A young man with a renal colic. J Intern Med. 2003;254(6):605-608.

6. Huang CC, Lo HC, Huang HH, Kao WF, Yen DH, Wang LM, Huang CI, et al. ED presentations of acute renal infarction. Am J Emerg Med. 2007;25(2):164-169.

7. Tsai SH, Chu SJ, Chen SJ, Fan YM, Chang WC, Wu CP, Hsu CW. Acute renal infarction: a 10-year experience. Int J Clin Pract. 2007;61(1):62-67.

8. Lopez VM, Glauser J. A case of renal artery thrombosis with renal infarction. J Emerg Trauma Shock. 
2010;3(3):302.

9. Poux JM, Boudet R, Lacroix P, Jauberteau MO, Plouin $\mathrm{PF}$, Aldigier JC, Leroux-Robert C. Renal infarction and thrombosis of the infrarenal aorta in a 35-year-old man with primary antiphospholipid syndrome. Am J Kidney Dis. 1996;27(5):721-725.

10. S YH, Shahgaldi K. Thrombo-embolic renal infarction in a case of mid-ventricular takotsubo syndrome. Intern Med. 2011;50(19):2175-2178.

11. McGee SM, Thompson CA, Granberg CF, Hutcheson JC, Vandersteen DR, Reinberg Y, Wolpert JJ. Acute renal infarction due to fungal vascular invasion in disseminated candidiasis. Urology. 2009;73(3):535-537.

12. Bolderman R, Oyen R, Verrijcken A, Knockaert D, Vanderschueren S. Idiopathic renal infarction. Am J Med. 2006;119(4):356 e359-312.

13. Braun DR, Sawczuk IS, Axelrod SA. Idiopathic renal infarction. Urology. 1995;45(1):142-145.

14. Paris B, Bobrie G, Rossignol P, Le Coz S, Chedid A, Plouin PF. Blood pressure and renal outcomes in patients with kidney infarction and hypertension. J Hypertens. 2006;24(8):1649-1654.

15. Yoshida T, Ikehara N, Miyabe H, Sakata S, Yajima K, Mukai S, Ohte N, et al. Two cases with renal infarction diagnosed in the early course using contrast-enhanced CT. Hypertens Res. 2004;27(7):523-526.

16. Fu ZF, Zhang ZG, Liu XM. A rare case of acute renal infarction due to idiopathic renal arterial thrombosis. Chin Med J (Engl). 2008;121(2):185-187.

17. Wong WS, Moss AA, Federle MP, Cochran ST, London SS. Renal infarction: CT diagnosis and correlation between CT findings and etiologies. Radiology. 1984;150(1):201-205.

18. Rhee H, Song SH, Won Lee D, Lee SB, Kwak IS, Seong EY. The significance of clinical features in the prognosis of acute renal infarction: single center experience. Clin Exp Nephrol. 2012;16(4):611-616.

19. Hazanov N, Somin M, Attali M, Beilinson N, Thaler M, Mouallem M, Maor Y, et al. Acute renal embolism. Forty-four cases of renal infarction in patients with atrial fibrillation. Medicine (Baltimore). 2004;83(5):292-299. 